\title{
ACCEDA, repositorio institucional de la ULPGC: mejoras y desarrollos propios
}

ACCEDA, institutional repository of ULPGC: developments and self-implementations

Ma Pilar LóPEZ DíEZ (1) Y Alejandro MeLIÁN VINUESA (2)

(1) Sección de Informatización. Biblioteca de la Universidad de Las Palmas de Gran Canaria. Edificio central de la Biblioteca Universitaria, Campus de Tafira, 35017 Las Palmas de Gran Canaria, plo-pez@pas.ulpgc.es (2) Nuevas Tecnologías de Canarias. Padre Dámaso Serna, n 1 local 5, 35011, Las Palmas de Gran Canaria, amv@ntcanarias.com

\section{Resumen}

Desde hace unos años, la Biblioteca de la Universidad de Las Palmas de Gran Canaria, está desarrollando iniciativas que promueven el acceso abierto entre sus usuarios. Fruto de este trabajo, se puso en marcha en el año 2009 el Repositorio Institucional ACCEDA (http://acceda.ulpgc.es). El software elegido para su desarrollo es DSpace. En el presente artículo se exponen las herramientas y los desarrollos propios creados e implementados para facilitar el trabajo de los administradores y el uso y consulta por parte de los usuarios. Entre las principales novedades incorporadas se incluyen la carga delegada, el control de autoridades y el desarrollo de una herramienta para material audiovisual en formato streaming.

Palabras clave: DSpace. Software libre. Repositorios institucionales. ACCEDA. Bibliotecas universitarias. Universidad de Las Palmas de Gran Canaria.

\section{Introducción}

El movimiento Open Access supone una nueva forma de entender el acceso a la literatura científica, ya que implica poder acceder al conocimiento sin las restricciones existentes en los sistemas de publicación tradicionales.

En los últimos años, el Open Access se ha desarrollado y expandido considerablemente, movido por el deseo de disminuir las barreras de acceso a la literatura científica. Como características principales podemos señalar; además de la gratuidad de los contenidos, la facilidad de acceso, la posibilidad de realizar búsquedas, la impresión y, no menos importante, la calidad de contenidos (Sanz-Valero, 2007).

Las instituciones, sobre todo las académicas, consideran un valor estratégico importantísimo la documentación generada por sus miembros, de ahí que sientan la necesidad de difundirla.

Hay que tener en cuenta que no sólo es esencial poner a disposición de toda la comunidad investigadora los trabajos e investigaciones,

\begin{abstract}
Over the last few years, the Library of the ULPGC has been developing initiatives that promote the free access amongst its users. In 2009 as a result of the work carried out until then, the Institutional Repository Acceda was launched (http://acceda.ulpgc.es). The software that was chosen for its development is DSpace. In this article we describe the tools and the developments especially created and implemented to facilitate the work of the administrators, as well as the usage and search function by the users. Some of the main innovations that have been incorporated are a batch item importer, authorities control and the development of a tool for audiovisual material streaming.
\end{abstract}

Keywords: DSpace. Open source. Institucional repositories. ACCEDA. Academic libraries. University of Las Palmas de Gran Canaria.

sino también asegurar su almacenamiento y preservación.

Como respuesta a estas nuevas necesidades surgen los repositorios institucionales, siendo las universidades y centros de investigación los principales impulsores de su desarrollo (Santillan-Aldana, 2009). Actualmente, los repositorios institucionales se han convertido en una herramienta básica para dar visibilidad, en formato digital, a la producción científica e institucional de una organización. Pero además, permiten organizarla, almacenarla y preservarla asegurando así su uso futuro. Las Nuevas Tecnologías de la Información son un aliado importantísimo en la consecución de este objetivo, ya que han facilitado el desarrollo de herramientas tanto de difusión como de almacenamiento y preservación.

\section{Proyectos iniciales}

La Biblioteca de la ULPGC (BULPGC) viene impulsando desde hace unos años iniciativas que promueven el acceso abierto a sus fondos, 
y desarrollando plataformas que ofrecen documentos a texto completo.

\subsection{Memoria Digital de Canarias}

Aunque los trabajos se iniciaron un año antes, en 2003 se puso en marcha la Biblioteca Digital, cuyo objetivo principal fue dar soporte a los proyectos de investigación de educación virtual, promoviendo el acceso a bases de datos científicas, revistas electrónicas, tesis doctorales, material multimedia y contenido de temática canaria.

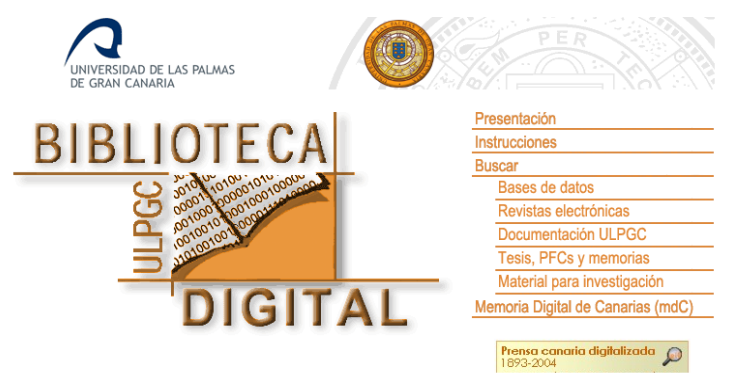

Figura 1. Portada de la Biblioteca Digital en su origen

Dentro de la Biblioteca Digital se creó la sección Memoria Digital de Canarias (mdC) (http://mdc. ulpgc.es) que, en su origen, supuso un proyecto inédito en el seno de una universidad española como una completa biblioteca regional en cuanto a contenidos locales, proporcionando siempre documentos completos. Este portal recoge documentación producida en Canarias, sobre $\mathrm{Ca}$ narias o de autores canarios de cualquier época, en cualquier tipo de soporte y actualmente cuenta con varias colecciones temáticas de gran importancia para la investigación del patrimonio canario.

\subsection{JABLE}

Otro proyecto de acceso abierto es JABLE (http://jable.ulpgc.es/jable/) que inicia su andadura en el año 2005 y se constituye como la principal plataforma de prensa canaria. JABLE fue en su origen el mayor repositorio de prensa digital en línea de España y ofrece acceso tanto a prensa histórica como viva, de temática o localización principalmente canaria, aunque también incorpora títulos nacionales y del África colonial española.

Actualmente se pueden consultar un total de 205 cabeceras y más de siete millones de páginas.

\section{ACCEDA: Repositorio Institucional}

El proyecto más reciente de acceso abierto iniciado por la BULPGC, es la creación del Repositorio institucional de la ULPGC, ACCEDA (http://acceda.ulpgc.es). Aunque ACCEDA se puso en marcha en octubre del 2009, su origen se remonta a la Biblioteca Digital, ya que en ella se creó una colección que ofrecía tesis y proyectos fin de carrera y cuyo objetivo era dar a conocer la documentación generada en la ULPGC.

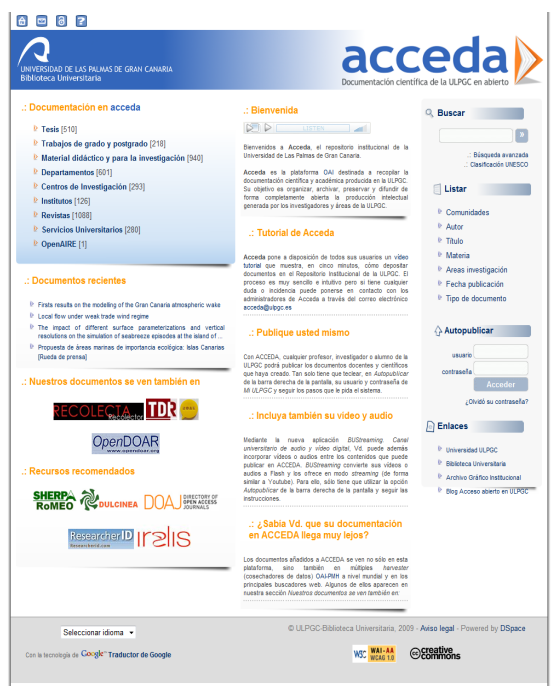

Figura 2. Portada de Acceda

Esta colección inicial del repositorio institucional fue poco a poco incluyendo más contenidos e incorporando los materiales utilizados para la docencia e investigación elaborados por el personal docente de la ULPGC.

Siguiendo esta línea de trabajo, se decidió crear una plataforma independiente para el repositorio institucional que recogiera tanto la documentación generada por la propia institución como la resultante de la investigación desarrollada en la ULPGC. Dicha plataforma se convierte así en el único punto de acceso a toda la producción científica e institucional de la ULPGC.

\subsection{Software}

El proyecto se inicia en 2006 con el estudio de las plataformas y los programas existentes en el mercado, tanto gratuitos como de sistema propietario. Entre todas las soluciones disponibles se analizaron DSpace, Greenstone, Eprints, Digitool y ContendtDM.

Teniendo en cuenta tanto el trabajo iniciado en los años anteriores como las necesidades, funcionalidad y servicios de la biblioteca digital se 
eligió DSpace, un software libre desarrollado por el Massachussets Institute of Technology (MIT) y la multinacional HP, que tiene como características más destacadas:

- Es un sistema de depósito digital dirigido principalmente a comunidades académicas que permite adaptarse a las necesidades de la institución posibilitando la creación de colecciones, comunidades...

- Permite el almacenamiento, preservación y redistribución de todo tipo de contenidos digitales (audio, vídeos, textos, imágenes...).

- Su uso está muy generalizado por un gran número de instituciones, principalmente académicas.

- Es software libre, lo que permite una gran autonomía y además cuenta con una comunidad de desarrolladores muy activa.

- Facilidad de manejo.

- Trabaja bajo protocolo OAl que permite ser cosechado por buscadores y recolectores científicos (OAlster, Recolecta, OpenDOAR, Google Scholar, etc)

\subsection{Desarrollos propios}

En 2009 tuvo lugar el lanzamiento de Acceda (Dspace versión 1.5.2) pero, sin duda, 2010 fue una fecha importante en cuanto a desarrollos técnicos. Durante este año se implementaron nuevas funcionalidades que facilitan su utilización por parte de los usuarios, pero también suponen una mejora significativa en los trabajos realizados por los administradores. Las herramientas desarrolladas surgen de la necesidad de dar respuesta a las demandas planteadas tanto por los usuarios como por los administradores. Una vez analizadas las soluciones existentes en el mercado, y puesto que no se encontraron aplicaciones que se adaptaran a nuestras necesidades, se decidió crear una serie de herramientas propias que facilitaran la gestión del repositorio.

\subsubsection{Diseño}

De las varias interfaces que tiene DSpace, se escogió trabajar con JSPUI por ser la más estable, ya que un gran número de repositorios trabajan con ella. El diseño se personalizó de tal manera que en la portada se incluyeron no sólo las comunidades del repositorio institucional, sino también los envíos recientes realizados, independientemente de la colección, así como enlaces a recursos de interés. Además, se creó un script para poder editar todos y cada uno de los bloques en los que está dividida la portada.

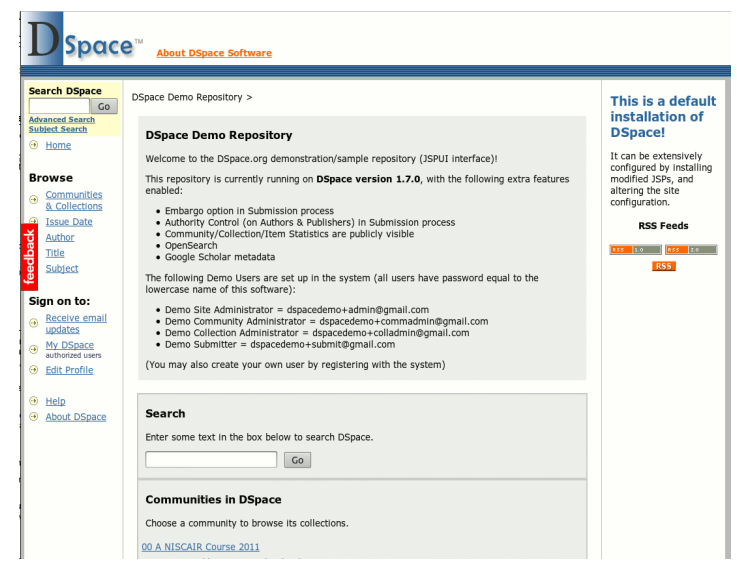

Figura 3. Imagen original de DSpace

La ficha simple del ítem posee un diseño muy cuidado y sobre todo funcional. Al entrar en ella, lo primero que destaca es la presentación de los metadatos tabulados y ordenados por colores según su tipo. Así, por ejemplo, el metadato que contiene el handle (1) se destaca en un color diferente del resto (naranja).

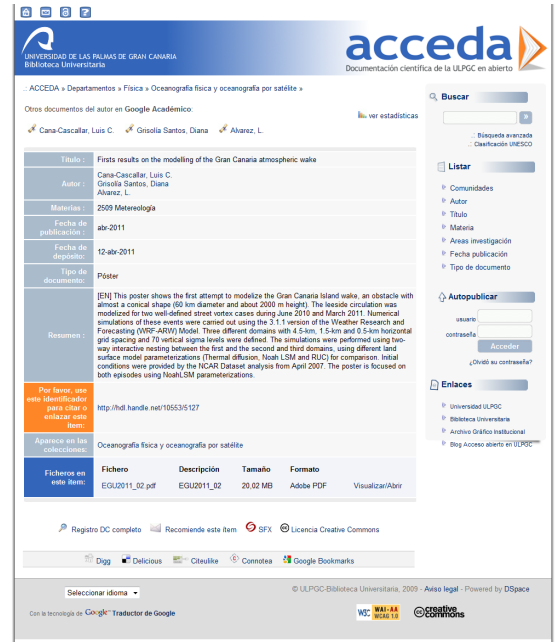

Figura 4. Registro del ítem sencillo en Acceda

Esto también ocurre con los objetos multimedia en streaming (2). Existe un visor integrado en la ficha simple donde se puede reproducir el objeto multimedia asociado de BUStreaming, funcionalidad que aumenta enormemente la usabilidad del repositorio.

Se añade además la posibilidad de buscar directamente en Google Académico las publicaciones de los autores del ítem sólo con pinchar en un enlace, así como enlazar el ítem a las 
redes sociales especializadas más populares como son Digg, Citeulike, Connotea o Google Bookmarks.

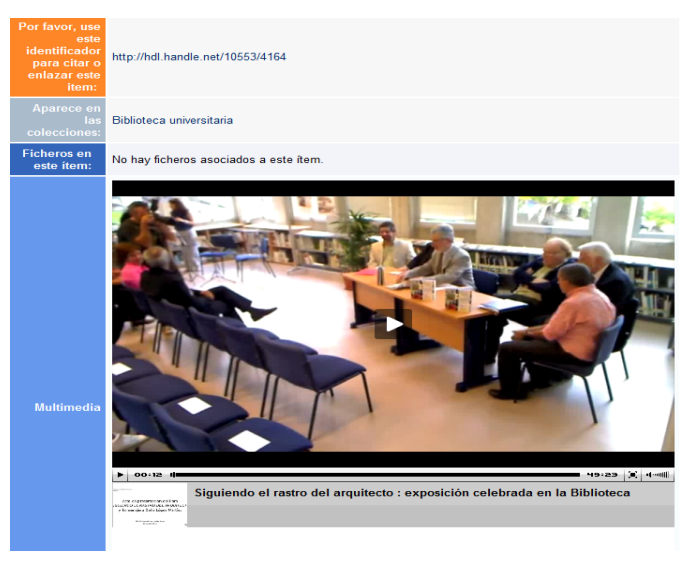

Figura 5. Registro multimedia

Para facilitar el proceso de depósito, se decidió incorporar en los primeros pasos de la autopublicación la Licencia de Depósito y las Licencias Creative Commons. En el caso de éstas últimas, se optó por integrar el formulario original de Creative Commons creándolo directamente en HTML para poder adecuarlo al diseño general del repositorio.

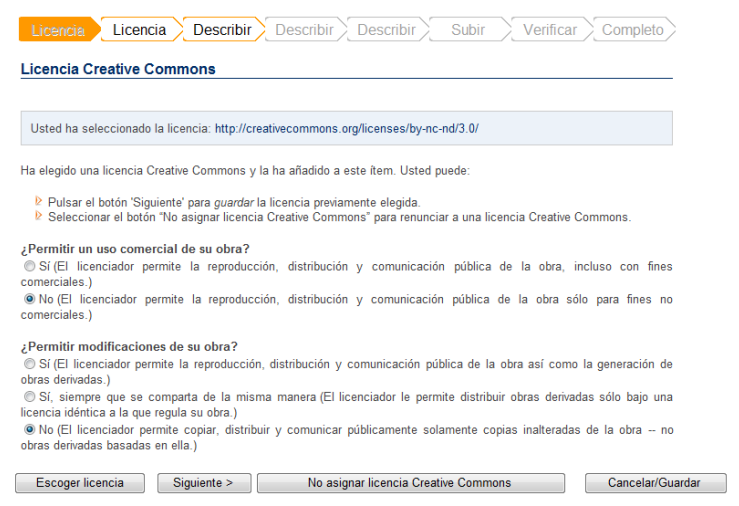

Figura 6. Creative Commons en auto-publicación

\subsubsection{Carga delegada}

Aunque la filosofía de Dspace es el auto-archivo (self-archiving), para impulsar la carga de documentos y facilitar a los usuarios esa tarea, se decidió diseñar una herramienta que permitiera delegar ese trabajo en la BU.

La idea fue crear una herramienta sencilla que permitiera al personal de la BU archivar en el repositorio las obras de los autores de la UL-
PGC que conserven los derechos de propiedad intelectual.

Dicha herramienta tiene una doble finalidad; por un lado, facilita a los usuarios el depósito de sus obras puesto que delegan esta tarea en el personal bibliotecario, lo que supone también un ahorro de tiempo y un impulso para potenciar el OA entre los investigadores; $y$, por otro, simplifica el trabajo del personal de la biblioteca en lo relativo a la carga de documentos.

Desde un principio se decidió que el diseño de la herramienta debía ser muy sencillo y, por supuesto, un reflejo de la auto-publicación, recogiendo todos y cada uno de los campos que esta aplicación ofrece al usuario para describir su obra.

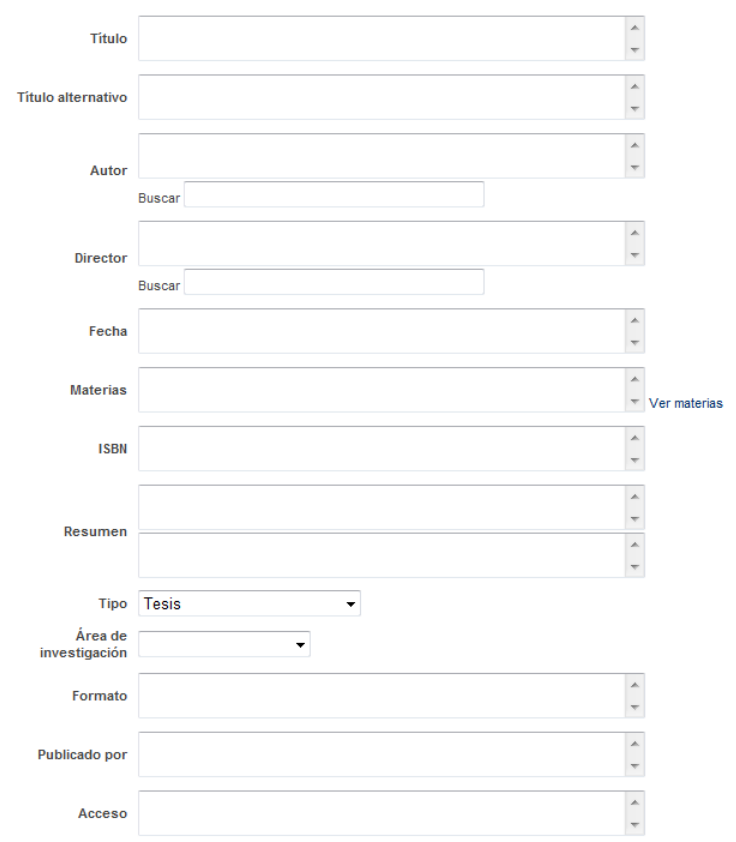

Figura 7. Formulario de carga delegada

Los tipos de cargas creados son dos: carga de ítem y carga masiva. La primera opción está destinada a la carga de ítems sueltos, mientras que la segunda se utiliza para realizar cargas masivas y poder incorporar al repositorio varios ítems a la vez, como por ejemplo, los artículos de las revistas del Servicio de Publicaciones de la ULPGC.

Para la implementación de la herramienta de carga delegada se hace uso del comando interno de DSpace para la importación de datos. Este comando debe ser usado desde la consola de comandos y se basa en parámetros, cuya utilización es laboriosa. 


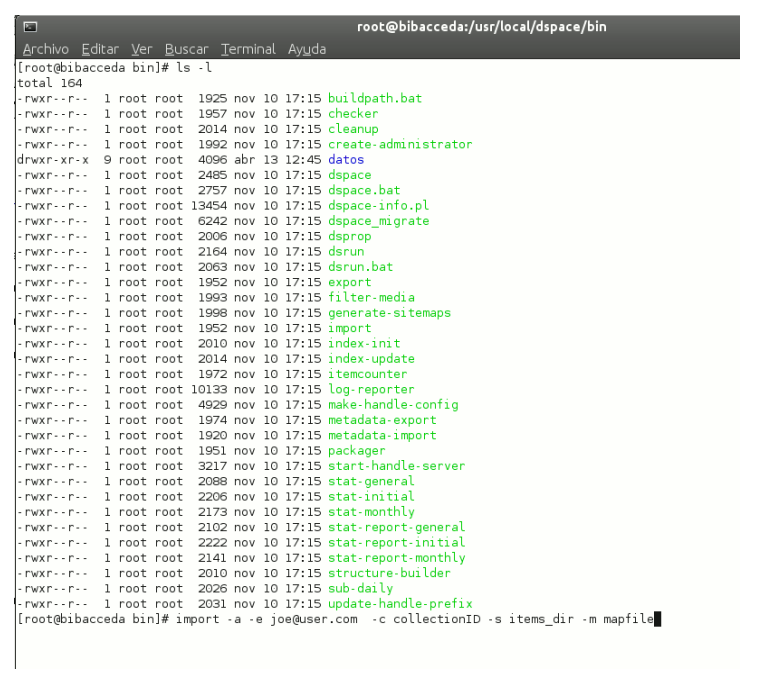

Figura 8. Consola de comandos

La carga delegada de Acceda intenta salvar esta brecha proporcionando una interfaz de usuario amigable. Es importante destacar el ahorro de tiempo que supone respecto a la auto-publicación.

Además de diseñar la herramienta hubo que articular un procedimiento para que el usuario siguiera teniendo el control sobre el depósito de su obra. Para ello y en colaboración con el Servicio Jurídico de la ULPGC, se elaboró una autorización que el usuario debe firmar y entregar en la biblioteca de su centro. En esta autorización se recogen tanto la licencia de depósito como las Licencias Creative Commons que están disponibles también en la autopublicación.

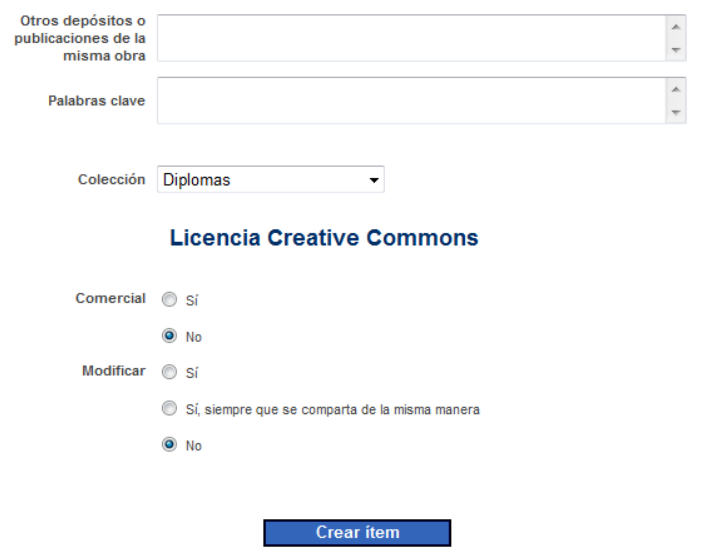

Figura 9. Creative Commons en el formulario de carga delgada

Para mejorar la gestión del administrador y llevar un control más exhaustivo, se ideó una herramienta que permitiera supervisar las cargas realizadas. A diferencia de la herramienta que incorpora las últimas versiones de Dspace, la aplicación desarrollada permite que los administradores puedan editar las cargas una vez preparadas y modificar los ítems en cualquier momento antes de que se lance la carga.

\subsubsection{Control de autoridades}

El cambio a la versión 1.6.2 realizado en 2010 se debió, entre otros factores, a la incorporación de nuevas funcionalidades importantes para el desarrollo del repositorio. Una de las grandes novedades que incluye esta nueva versión es el control de autoridades.

El control de autoridades es un elemento esencial para el repositorio, puesto que ayuda a establecer una forma única para los puntos de acceso de autor, asegurando así la coherencia y permitiendo la recuperación de todos los trabajos de un mismo autor. Es fundamental establecer un control de autoridades apropiado al sistema con el que se está trabajando, ya que optimiza considerablemente la recuperación de la información y además permite evitar duplicidades y ruido en las búsquedas.

La versión 1.6.2 incorpora un sistema de control basado en las autoridades de la Library of Congress, motivo por el cual hubo que buscar otra solución que se ajustara más a nuestras necesidades y a las características y filosofía del auto-archivo. Entre las diversas soluciones que se barajaron estaba la de incorporar en Acceda las autoridades del catálogo de la BULPGC, pero pronto se desestimó esta posibilidad, porque en el catálogo se trabaja bajo las directrices de las Reglas de catalogación, lo que impediría al usuario elegir la forma en la que quiere ser citado.

Por otro lado, hay que tener en cuenta también que la visibilidad de los repositorios institucionales es mucho mayor que la que puede tener un catálogo local, puesto que éstos son cosechados por grandes recolectores, ampliando enormemente la visibilidad de la documentación archivada en ellos. A todo lo expuesto hasta aquí hay que añadir la cuestión lingüística. Debemos considerar que, en el ámbito anglosajón, no se tienen en cuenta los dos apellidos, ni los nombres del mundo hispano. Por último, las normas de publicación de algunas revistas científicas marcan pautas en cuanto a la redacción de los nombres de los autores.

Por todos los motivos expuestos y para no obligar al usuario a elegir una forma determinada para la redacción de su nombre, se decidió utilizar la propia base de datos de autoridades de Acceda basándose en el uso de un control 
AJAX (3) que muestra un listado de las autoridades existentes a partir de la introducción del cuarto carácter.

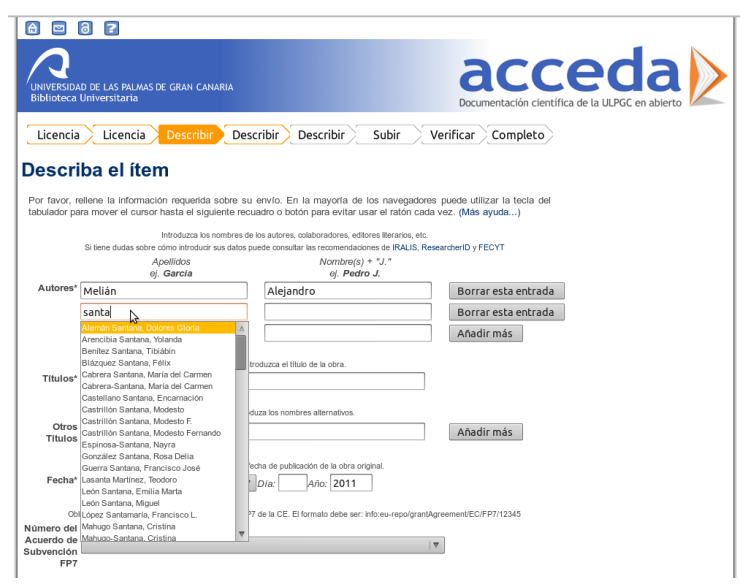

Figura 10. Control de autoridades en la auto-publicación

Para orientar a los autores se han puesto a su disposición enlaces a las recomendaciones de la FECYT y a recursos como IRALIS y ResearcherID. El sistema es abierto, puesto que cada vez que se archiva un documento con una autoridad nueva, ésta pasa a formar parte de la base de datos.

La creación del control de autoridades no sólo se desarrolló en el sistema de autoarchivo sino que también se implementó en la herramienta de carga delegada.

\subsubsection{BUstreaming}

En 2009 se desarrolló una herramienta propia que permite incorporar en Acceda y mdC material multimedia. La idea inicial fue crear un sistema que ofreciera material de audio y vídeo en formato streaming. Para la creación de esta herramienta se contó con el Servicio de Informática de la ULPGC.

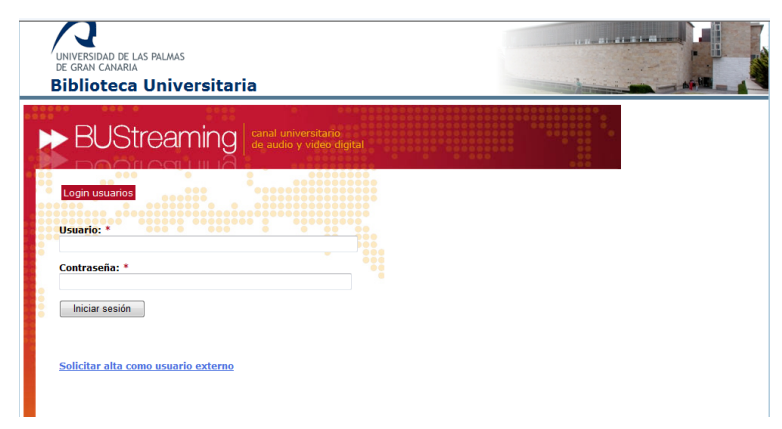

Figura 11. Imagen de BUstreaming
Una vez creada, se decidió incorporarla en el repositorio de manera sencilla e invisible al usuario para que éste pudiera añadir sin problemas sus vídeos y audios.

En la autopublicación de Acceda se añadió un nuevo paso donde el usuario puede introducir cómodamente sus objetos multimedia procedentes de BUStreaming. Para evitar que tuviera que copiar el código HTML y luego pegarlo en Acceda se creó un servicio web (4) como pasarela desde Acceda a BUStreaming y viceversa. Sin salir de Acceda se puede entrar en BUStreaming, seleccionar el objeto multimedia y continuar con la auto-publicación en Acceda teniendo ya el código HTML del objeto multimedia en el ítem.

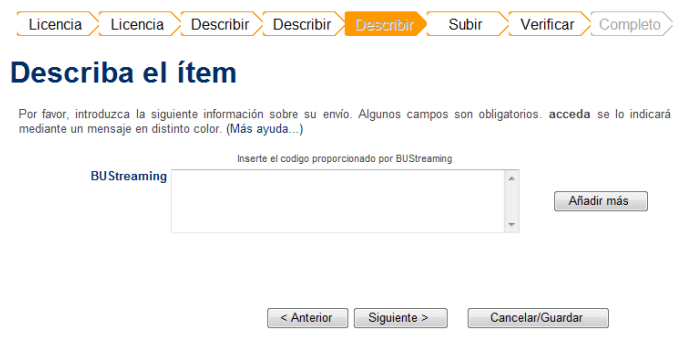

Figura 12. BUstreaming en la auto-publicación

\section{Acceda en cifras}

La puesta en marcha de Acceda se hizo con documentos en acceso abierto, procedentes de $\mathrm{mdC}$, en concreto tesis, proyectos de fin de carrera y material docente, sumando un total de 2.256 ficheros.

En este año y medio (tabla I), la promoción del Repositorio institucional entre los usuarios y el personal de biblioteca, así como las herramientas de-sarrolladas, han influido positivamente en su crecimiento tal y como se refleja en las estadísticas proporcionadas por Dspace. Hay que tener en cuenta que en el año 2009 sólo se han contabilizado los dos últimos meses puesto que el repositorio se puso en funcionamiento en el mes de octubre.

La principal aportación para este incremento ha sido la utilización del sistema de carga delegada. El diseño de la herramienta y su puesta en marcha se realizó durante el año 2010, y su utilidad es tal que, en ese año, el $92,1 \%$ de los documentos nuevos que se depositaron en Acceda fue mediante carga delegada. Este incremento tan significativo de documentos se debe principalmente a la incorporación de las revistas editadas por el Servicio de Publicaciones y Difusión Científica de la ULPGC, colaboración que 
se sigue manteniendo en la actualidad y que permite poner en acceso abierto publicaciones como: El Guiniguada, Vegueta, Philologica Canariensia y Espejo de paciencia entre otras.

\begin{tabular}{llll}
\hline & 2009 & 2010 & 2011 \\
\hline Items totales & 95 & 1684 & 611 \\
\hline Auto-archivo & 95 & 113 & 55 \\
\hline Descargas & 54111 & 517698 & 765133 \\
\hline Búsquedas & 5856 & 62244 & 56534 \\
& & & \\
\hline Sesiones iniciadas & 351 & 1440 & 630 \\
\hline
\end{tabular}

Tabla I. Estadísticas

En 2009 sólo estaban disponibles 222 artículos, mientras que ahora podemos consultar un total de 1.331 artículos pertenecientes a 10 revistas que cubren principalmente las áreas de Humanidades y Ciencias Sociales. La carga de todo este material recae en los administradores del repositorio, que utilizan la opción de la carga masiva por ser el sistema con el que se ahorra más tiempo, ya que permite subir en un mismo proceso varios números de la misma revista.

El depósito de documentos nuevos, sigue teniendo un balance positivo, puesto que a finales del mes de junio del año en curso, el 90 ' $8 \%$ de los documentos nuevos se han archivado mediante la carga delegada.

Aunque estamos en la primera mitad del año, los datos estadísticos hasta la fecha indican que el uso del Repositorio institucional va creciendo a buen ritmo. El número de sesiones iniciadas por usuarios registrados ha crecido así como las búsquedas y las descargas.

\section{Líneas futuras}

El repositorio institucional está en continuo desarrollo y crecimiento. Desde la BULPGC se sigue trabajando para implementar nuevas aplicaciones y contenidos que den respuesta a las nuevas necesidades que van surgiendo.
Siguiendo la línea de trabajo planteada hasta el momento, se siguen desarrollando nuevas funcionalidades y mejorando las existentes como por ejemplo: la edición masiva de metadatos, las búsquedas en el histórico de cargas y la incorporación de nuevos tipos de contenidos.

\section{Notas}

(1) El sistema Handle ofrece un servicio de resolución eficiente y seguro para la identificación unívoca y persistente de objetos digitales. La dependencia de dominios y direcciones IP es inexistente, ya que el sistema redirecciona al usuario a cualquiera que sea su ubicación, ya que se le asigna un identificador único para siempre.

(2) El streaming consiste en la distribución de audio o vídeo por Internet. La palabra streaming se refiere a que se trata de una corriente continua (sin interrupción). El usuario puede escuchar o ver en el momento que quiera. Este tipo de tecnología permite que se almacenen en un búfer lo que se va escuchando o viendo. El streaming hace posible escuchar música o ver vídeos sin necesidad de ser descargados previamente.

(3) Ajax, acrónimo de Asynchronous JavaScript And XML (JavaScript asíncrono y $\mathrm{XML}$ ), es una técnica de desarrollo web para crear aplicaciones interactivas o RIA (Rich Internet Applications). Estas aplicaciones se ejecutan en el cliente, es decir, en el navegador de los usuarios, mientras se mantiene la comunicación asíncrona con el servidor en segundo plano. De esta forma es posible realizar cambios sobre las páginas sin necesidad de recargarlas, lo que significa aumentar la interactividad, velocidad y usabilidad de las aplicaciones.

(4) Un servicio web (en inglés, Web service) es un conjunto de protocolos y estándares que sirven para intercambiar datos entre aplicaciones. Distintas aplicaciones de software -desarrolladas en lenguajes de programación diferentes y ejecutadas sobre cualquier plataforma- pueden utilizar los servicios web para intercambiar datos en redes de ordenadores como Internet. La interoperabilidad se consigue mediante la adopción de estándares abiertos.

\section{Referencias}

Santillan-Aldana, Julio (2009). El movimiento del acceso abierto y el mundo bibliotecario desde la experiencia del proyecto E-LIS. // Revista AIBDA. 30:1-2. www.cnpt.em brapa.br/RevistaAIBDA/v30/v30n1d02.pdf (20-06-2011).

Sanz-Valero, J.; D'Agostino, M. J.; Castiel, L. D.; Veiga de Cabo, J. (2007). La iniciativa open access, una visión de conjunto. // Med. Segur. Trab. 53:207, 5-10. http://hdl.handle.net/10760/12213 (21-02-2011). 
